\title{
Breastfeeding and occlusal development
}

\author{
Abstracted from \\ Boronat-Catalá M, Montiel-Company JM, Bellot-Arcís C, Almerich-Silla JM, Catalá-Pizarro M.
}

Association between duration of breastfeeding and malocclusions in primary and mixed dentition: a systematic review and meta-analysis. Sci Rep 2017; 7: 5048. doi:10.1038/s41598-017-05393-y. PubMed PMID: 28698555; PubMed Central PMCID: PMC5505989.

Address for correspondence: Montserrat Boronat-Catalá, Department of Stomatology, Faculty of Medicine and Dentistry, University of Valencia, Valencia, Spain. E-mail: Montse_boronat@hotmail.com

\section{Question: Does breastfeeding affect malocclusions in primary and mixed dentitions?}

Data sources Pubmed, Embase, Scopus databases and New York Academy of Medicine Grey Literature Report. There were no restrictions on language and publication dates.

Study selection Two reviewers selected both prospective and retrospective studies of children who had either been exclusively breastfed or were mixed breastfed; who were in either the primary or mixed dentition, and malocclusion was assessed. The following malocclusion traits were included; Class 2 molar, open-bite, non-spaced dentition and posterior crossbite.

Data extraction and synthesis Data were extracted including type of dentition, type of malocclusion, breastfeeding data collection method, results, breast feeding malocclusion odds ratio (OR) and quality of the article. The ORs of associations between different lengths of breastfeeding and the various malocclusion traits were calculated. Results Thirty-one studies were included in a qualitative synthesis, nine in the quantitative analysis. All studies were observational, a majority examining malocclusion in the mixed dentition. Two studies looked at associations between no breastfeeding and posterior crossbite, finding children not breastfed presented 1.7 times more posterior crossbite than those breastfed for between one and six months $(\mathrm{OR}=1.70$ $\mathrm{Cl}$ 2.01-2.39). ORs were calculated for exclusive breastfeeding and posterior crossbite with different duration of breastfeeding, and duration of breastfeeding and posterior crossbite. With regard to open bite and breastfeeding the $\mathrm{OR}=1.76(\mathrm{Cl} 0.55-5.61)$ comparing those who were breastfed for less than or more than six months. Children breastfed for up to six months presented 1.25 times more Class 2 molar relationships than those breastfed for over six months. Children breastfed for up to six months presented 1.73 times more non-spaced dentitions than those breastfed for over six months ( $\mathrm{OR}=1.73, \mathrm{Cl} 1.35-2.22$ ).

Conclusions Breastfeeding is a protective factor against posterior crossbites and Class 2 malocclusion in primary and mixed dentitions, with the protective effect increasing with the months of breastfeeding. There is no clear evidence for breastfeeding proving any benefit against other malocclusion risks; open bite and non-spaced dentitions.

\section{Commentary}

In the UK, more than $73 \%$ of mothers start breastfeeding, ${ }^{1}$ with the WHO recommending exclusive breastfeeding up to six months of age. ${ }^{2}$ It is thought that breastfeeding can promote better occlusal development but there is dispute within the literature. The aim of this review was to look at the evidence on the possible effects of breastfeeding (BF) on different malocclusion traits in the primary and mixed dentition.

An extensive database search was carried out with no restriction on language or publication date. Both prospective and retrospective studies were included; with randomised controlled trials, case-control studies and cohort studies reviewed. Thirty-one observational studies were included in the qualitative analysis, and nine in the meta-analysis. It is not stated if data extraction was carried out independently and by whom. The quality of the studies was assessed using the Newcastle Ottawa Scale independently by two reviewers and was found to be moderate to high for all studies. Odds ratios were calculated for associations between different lengths of BF and different malocclusion traits and where studies were combined weighted OR was calculated.

A detailed summary of qualitative information was given with the following observations; posterior crossbite is more prevalent in the primary and mixed dentition in children who had received no breastfeeding or BF for up to six months. Open bites are more common in children with no BF or only a few months of BF. The evidence was mixed in support of BF reducing Class 2 malocclusions, improving occlusal development and aiding spaced dentitions.

There were multiple results from the quantitative analyses, with the main ones being; children who were not BF had an increased chance of posterior crossbite, this chance increased with prolonged BF. There was no association between BF duration and open bite. Children who were BF for more than six months had less Class 2 relationships, but were less likely to have spaced dentitions.

The authors recognise the limitations of the results of this systematic review, due in large to the types of studies identified. They are aware of the possible recall bias of parents regarding lengths of time children were BF, if BF was exclusive or the possibility of a confounding issue such as use of a pacifier. Due to the heterogeneity of the studies comparability was difficult. The authors acknowledge the need for prospective studies, eliminating any possible confounders. However, they do conclude that $\mathrm{BF}$ is a protective factor against posterior crossbites and Class 2 malocclusions in primary and mixed dentition, with the protective effect increasing with months of BF. This is a bold statement to make in view of the limited low level of evidence. Also, it would be interesting to know how many children go on to develop a crossbite in the permanent dentition as this is when the majority of orthodontic treatment occurs in the UK. Does the 'protective factor' wear off?

Although the methodology of the systematic review is sound, few conclusions should be drawn from it.

Felicity Borrie

Consultant Orthodontist, Dr Gray's Hospital, Elgin, Scotland

1. https://www.nhs.uk/conditions/pregnancy-and-baby/benefits-breastfeeding/ (accessed March 2018)

2. http://www.who.int/topics/breastfeeding/en/ (accessed March 2018) 